\title{
THE GENERALISED LIÉNARD EQUATIONS
}

\author{
A. AGHAJANI \\ School of Mathematics, Iran University of Science and Technology, Tehran, Iran \\ e-mail:aghajani@iust.ac.ir \\ and A. MORADIFAM \\ Department of Mathematics, University of British Columbia, Vancouver BC, Canada \\ e-mail:a.moradi@math.ubc.ca
}

(Received 3 September 2007; accepted 15 May 2009)

\begin{abstract}
In this paper we present sufficient conditions for all trajectories of the system

$$
\dot{x}=\frac{1}{a(x)}[h(y)-F(x)], \quad \dot{y}=-a(x) g(x),
$$

to cross the vertical isocline $h(y)=F(x)$, which is very important in the global asymptotic stability of the origin, oscillation theory and existence of periodic solutions. Also we give sufficient conditions for all trajectories which start at a point on the curve $h(y)=F(x)$, to cross the $y$-axis which is closely connected with the existence of homoclinic orbits, stability of the zero solution, oscillation theory and the centre problem. The obtained results extend and improve some of the authors' previous results and some other theorems in the literature.
\end{abstract}

2000 Mathematics Subject Classification. 34C37, 34D05, 34C05.

1. Introduction. We consider the Liénard-type system

$$
\dot{x}=\frac{1}{a(x)}[h(y)-F(x)], \quad \dot{y}=-a(x) g(x),
$$

where $a(x)>0$ for every $x \in \mathbb{R}$ and $h(y)$ is continuous and strictly increasing on $\mathbb{R}$. The functions $F(x), g(x), a(x)$ and $h(y)$ satisfy smoothness conditions for uniqueness of solutions of initial value problems. Also we assume that all solutions of this system are continuable in the future time. Throughout this paper, we assume that $F(0)=0$, $h( \pm \infty)= \pm \infty$,

$$
x g(x)>0 \text { for } x \neq 0 \text { and } y h(y)>0 \text { for } y \neq 0,
$$

which guarantee that the origin is the unique equilibrium of system (1.1). Let $h^{-1}(w)$ be the inverse function of $w=h(y)$. Notice also that $h^{-1}(w)$ is strictly increasing and satisfies $w h^{-1}(w)>0$ for $w \neq 0$.

System (1.1) and some of its special cases have been widely studied by many authors, and the results can be found in many books and papers [1-25]. We say that system (1.1) has property $\left(X^{+}\right)$in the right half-plane (resp. in the left half-plane) if for every point $\left(x_{0}, y_{0}\right)$ with $h\left(y_{0}\right)>F\left(x_{0}\right)$ and $x_{0} \geq 0\left(\right.$ resp. $h\left(y_{0}\right)<F\left(x_{0}\right)$ and $\left.x_{0} \leq 0\right)$, the 
positive semi-trajectory of (1.1) passing through $\left(x_{0}, y_{0}\right)$ crosses the vertical isocline $h(y)=F(x)$. Also system (1.1) is said to have property $\left(X^{-}\right)$in the right half-plane (resp. in the left half-plane), if for every point $\left(x_{0}, y_{0}\right)$ with $h\left(y_{0}\right)<F\left(x_{0}\right)$ and $x_{0} \geq 0$ (resp. $h\left(y_{0}\right)>F\left(x_{0}\right)$ and $\left.x_{0} \leq 0\right)$, the negative semi-trajectory of (1.1) passing through $\left(x_{0}, y_{0}\right)$ crosses the vertical isocline $h(y)=F(x)$.

We say that system (1.1) has property $\left(Y^{+}\right)$in the right half-plane (resp. in the left half-plane) if for every point $P\left(x_{0}, y_{0}\right)$ with $F\left(x_{0}\right)=h\left(y_{0}\right)$ and $x_{0}>0$ (resp. $\left.x_{0}<0\right)$ the positive semi-trajectory of (1.1) starting at $P$ intersects the negative $y$-axis (resp. positive $y$-axis). We also say that system (1.1) has property $\left(Y^{-}\right)$in the right half-plane (resp. in the left half-plane) if for every point $P\left(x_{0}, y_{0}\right)$ with $F\left(x_{0}\right)=h\left(y_{0}\right)$ and $x_{0}>0$ (resp. $\left.x_{0}<0\right)$ the negative semi-trajectory of $(1.1)$ starting at $P$ intersects the positive $y$-axis (resp. negative $y$-axis). Properties $\left(X^{+}\right)$and $\left(X^{-}\right)$have been widely studied by many authors (see $[\mathbf{1}-\mathbf{2}, \mathbf{6}, \mathbf{8}, \mathbf{1 1}-\mathbf{1 2}, \mathbf{2 1})$ because they are fundamental concepts in the existence of periodic solutions, oscillation theory and global asymptotic stability of the zero solution.

Define

$$
G(x)=\int_{0}^{x} a^{2}(\eta) g(\eta) d \eta .
$$

Recently, in [1] the authors proved the following theorem which includes all previous sufficient conditions for property $\left(X^{+}\right)$for the classical Liénard system as special cases.

Theorem A. Assume $G(+\infty)=+\infty$. Then system $(1.1)$ with $a(x)=1$ and $h(y)=y$ has property $\left(X^{+}\right)$in the right half-plane if

$$
\limsup _{x \rightarrow+\infty}\left(\int_{b}^{x}\left(\frac{F(\eta) g(\eta)}{(2 G(\eta))^{\frac{3}{2}}}+\frac{g(\eta)}{G(\eta)}\right) d \eta+\frac{F(x)}{\sqrt{2 G(x)}}\right)=+\infty,
$$

for some $b>0$.

Also, in [2] the authors presented an implicit necessary and sufficient condition for property $\left(X^{+}\right)$in the Liénard plane. Using this condition we derived explicit conditions and solved the problem of intersection with the vertical isocline in the Lienard plane completely in some sense. These results answered an old conjecture of Hara and Sugie [10] as well. The problem is much more difficult for the generalised equation (1.1). In [8] we have a nice result.

Theorem B. Assume that $h( \pm \infty)= \pm \infty$ and $\limsup _{x \rightarrow+\infty} F(x)>-\infty$. Then system (1.1) has property $\left(X^{+}\right)$in the right half-plane if and only if

$$
\limsup _{x \rightarrow+\infty}\left[\int_{0}^{x} \frac{a^{2}(\eta) g(\eta)}{1+F_{-}(\eta)} d \eta+F(x)\right]=+\infty,
$$

where $F_{-}(x)=\max \{0,-F(x)\}$.

On the other hand a necessary and sufficient condition for property $\left(X^{+}\right)$in the right half-plane is not yet obtained under the assumption

$$
\lim _{x \rightarrow+\infty} F(x)=-\infty
$$


In [8] Gyllenberg and Ping generalised the results of Jiang [14] and Yan and Jiang [22] by the following theorem.

TheOrem C. Assume that $h(-\infty)=-\infty$ and $\lim _{x \rightarrow+\infty} F(x)=-\infty$. If there exist $\beta>\frac{1}{4}$ and $N_{1}>0$ such that $F(x)<0$ for $x \geq N_{1}$, and iffor every fixed $k \geq 1$ and $b \geq N_{1}$, there exists $\bar{b}>b$ satisfying

$$
\int_{b}^{x} \frac{a^{2}(\eta) g(\eta)}{F(\eta)} d \eta \leq \frac{1}{k} h^{-1}(k \beta F(x)) \quad \text { for } x \geq \bar{b},
$$

then system (1.1) has property $\left(X^{+}\right)$if and only if (1.4) in Theorem B holds.

In [12] one of the authors presented an implicit necessary and sufficient condition for property $\left(X^{+}\right)$in the generalised Liénard equations and proved very sharp explicit sufficient and necessary conditions.

Many authors have also investigated property $\left(Y^{+}\right)$, and several interesting sufficient conditions have been given. In [8] Gyllenberg and Ping presented a sufficient condition for system (1.1) to have property $\left(Y^{+}\right)$. Hara and Yoneyama in [7] proved that if there exists an $a>0$ such that $F(x)>0$ for $0<x \leq a$ and some $\alpha>\frac{1}{4}$ such that

$$
\frac{1}{F(x)} \int_{0}^{x} \frac{g(\eta)}{F(\eta)} d \eta \geq \alpha,
$$

then system $(1.1)$ with $a(x)=1$ and $h(y)=y$ has property $\left(Y^{+}\right)$(see also $\left.[\mathbf{3}, \mathbf{6}, \mathbf{1 3}, \mathbf{2 0}]\right)$. Recently authors proved some sufficient and necessary conditions for property $\left(Y^{+}\right)$ in system (1.1), which are very sharp [3].

In this paper we extend Theorem A for the generalised Liénard equation (1.1). Also, we will use the same idea to prove analogous results for system (1.1) to have property $\left(\mathrm{Y}^{+}\right)$. The obtained results include several sufficient conditions presented in the previous literature as special cases. By some examples we show that our results are applicable when none of the results presented above are applicable. Similar results can be formulated for properties $\left(X^{-}\right)$and $\left(Y^{-}\right)$.

2. Sufficient conditions for property $\left(X^{+}\right)$. In this section we intend to present some sufficient conditions for system (1.1) to have property $\left(X^{+}\right)$in the right halfplane. Notice that under the assumption $\lim \sup _{x \rightarrow+\infty} F(x)>-\infty$, Theorem B gives a necessary and sufficient condition for system (1.1) to have property $\left(X^{+}\right)$in the right half-plane. Hence, throughout this section we assume that

$$
\lim _{x \rightarrow+\infty} F(x)=-\infty
$$
$\left(X^{+}\right)$.

The following lemma gives a necessary condition for system (1.1) to have property

LEMMA 2.1. Suppose that (2.1) holds. If system (1.1) has property $\left(X^{+}\right)$in the right half-plane, then

$$
\int_{0}^{\infty} \frac{a^{2}(\eta) g(\eta)}{1+F_{-}(\eta)} d \eta=+\infty
$$


Proof. By (2.1) there exist $x_{0}>0$ and $M>0$ such that $F(x) \leq 0$, for $x \geq x$, and $M \geq h^{-1}(F(x))$, for every $x>0$. Since, $h(+\infty)=+\infty$, we can choose $M^{\prime}>M$ such that $h(y) \geq \operatorname{Max}\{1, M\}$, for every $y \geq M^{\prime}$. Now let $n \in \mathbb{N}$ and consider the solution $(x(t), y(t))$ of $(1.1)$ with $(x(0), y(0))=\left(x_{0}, M^{\prime}+n\right)$. Notice that while $(x(t), y(t))$ lies in the region $\left\{(x, y) \in \mathbb{R}^{2}: x \geq 0\right.$ and $\left.h(y)>F(x)\right\}$ we have $\dot{x}>0$ and $\dot{y}<0$. Since, system (1.1) has property $\left(X^{+}\right)$in the right half-plane, this solution must cross the curve $h(y)=F(x)$. Thus, there exists a $t_{n}>0$ such that $y\left(t_{n}\right)=M^{\prime}$ and $M^{\prime} \leq y(t) \leq M^{\prime}+n$, for $0 \leq t \leq t_{n}$. Moreover, we have $x(t) \geq x_{0}$ for $0 \leq t \leq t_{n}$. Hence, $F(x(t)) \leq 0$ for $t>0$ and

$$
h(y(t))-F(x(t)) \geq \operatorname{Max}\{1, M\}-F(x(t)) \geq 1+F_{-}(x(t)),
$$

for $0 \leq t \leq t_{n}$. Now we have

$$
\begin{aligned}
n & =y(0)-y\left(t_{n}\right)=\int_{0}^{t_{n}} \frac{a^{2}(x(\eta)) g(x(\eta))}{h(y(\eta))-F(x(\eta))} \dot{x}(\eta) d \eta \\
& \leq \int_{0}^{x\left(t_{n}\right)} \frac{a^{2}(\eta) g(\eta)}{1+F_{-}(\eta)} d \eta<\int_{0}^{\infty} \frac{a^{2}(\eta) g(\eta)}{1+F_{-}(\eta)} d \eta .
\end{aligned}
$$

Thus, (2.2) holds and the proof is complete.

Lemma 2.2. Suppose that (2.2) holds and $(x(x), y(t))$ is a solution of (1.1) which starts at a point $\left(x_{0}, y_{0}\right) \in\left\{(x, y) \in \mathbb{R}^{2}: x \geq 0\right.$ and $\left.h(y)>F(x)\right\}$ and does not cross the curve $h(y)=F(x)$. Then

$$
\lim _{t \rightarrow \infty} y(t)=-\infty
$$

Proof. Notice that

$$
\begin{aligned}
y(t)-y_{0} & =-\int_{0}^{t} a(x(\eta)) g(x(\eta)) d \eta-\int_{0}^{t} \frac{a^{2}(x(\eta)) g(x(\eta))}{h(y(\eta))-F(x(\eta))} \dot{x}(\eta) d \eta \\
& =-\int_{x_{0}}^{x(t)} \frac{a^{2}(\eta) g(\eta)}{h(y(\eta))-F(\eta)} d \eta \\
& \leq-\int_{x_{0}}^{x(t)} \frac{a^{2}(\eta) g(\eta)}{h(y(0))+F_{-}(\eta)} d \eta \rightarrow-\infty,
\end{aligned}
$$

as $t \rightarrow+\infty$. The proof is complete.

Now suppose that there exists a solution $(u(t), v(t))$ of (1.1) whose graph remains in the region $\{(u, v): u \geq 0$ and $h(v)>F(u)\}$ for all future time. Let $\left(u_{0}, v_{0}\right)=(u(0), v(0))$. Since, system (1.1) has no critical points in this region and $\dot{u}>0$, we have

$$
u(t) \rightarrow+\infty \text { as } t \rightarrow+\infty
$$

so, we may assume that $u_{0}$ is sufficiently large and

$$
u(t) \geq u_{0}>0 \text { for } t>0
$$


Now let

$$
\chi(t)=\int_{b}^{u(t)} \frac{a^{2}(s) F(s) g(s)}{(2 G(s)) \frac{3}{2}} d s+\frac{K(v(t))}{\sqrt{2 G(u(t))}},
$$

where $K(y)$ is a continuously differentiable function such that $K^{\prime}(y)>0$ and $K(y) \geq$ $h(y)$, for $y<0$, with $|y|$ sufficiently large. Then

$$
\begin{aligned}
\dot{\chi}(t)= & \dot{u}(t) \frac{a^{2}(u(t)) F(u(t)) g(u(t))}{(2 G(u(t))) \frac{3}{2}} \\
& -\frac{2 a(u(t)) g(u(t)) G(u(t)) K^{\prime}(v(t))+a^{2}(u(t)) g(u(t)) K(v(t)) \dot{u}(t)}{(2 G(u(t))) \frac{3}{2}} .
\end{aligned}
$$

If (2.2) holds, then by Lemma 2.2 we get

$$
\begin{aligned}
\dot{\chi}(t) & \leq-\frac{2 a(u(t)) g(u(t)) G(u(t)) K^{\prime}(v(t))+a^{3}(u(t)) g(u(t))(\dot{u}(t))^{2}}{(2 G(u(t))) \frac{3}{2}} \\
& \leq-\frac{a^{2}(u(t)) g(u(t)) \sqrt{K^{\prime}(v(t))}}{G(u(t))} \dot{u}(t)
\end{aligned}
$$

fot $t>0$ sufficiently large. Hence,

$$
\dot{\chi}(t) \leq-\frac{a^{2}(u(t)) g(u(t)) \sqrt{K^{\prime}(v(t))}}{G(u(t))} \dot{u}(t),
$$

for $t>0$ sufficiently large.

TheOrem 2.1. Assume (2.1) and (2.2) hold and $h(y) \leq m y$, for $y<0$, with $|y|$ sufficiently large and some $m>0$. Then system (1.1) has property $\left(X^{+}\right)$in the right half-plane if

$$
\limsup _{x \rightarrow+\infty}\left(\int_{b}^{x}\left(\frac{a^{2}(\eta) F(\eta) g(\eta)}{(2 G(\eta))^{\frac{3}{2}}}+\frac{\sqrt{m} a^{2}(\eta) g(\eta)}{G(\eta)}\right) d \eta+\frac{F(x)}{\sqrt{2 G(x)}}\right)=+\infty,
$$

for some $b>0$.

Proof. We prove the theorem by contradiction. Suppose that system (1.1) fails to have property $\left(X^{+}\right)$in the right half-plane. Then there exists a solution $(u(t), v(t))$ of (1.1) whose graph remains in the region $\{(u, v): u \geq 0$ and $h(v)>F(u)\}$ for all future time. Let $K(y)=m y$. Thus, from (2.4) we have

$$
\frac{d}{d t}\left(\chi(t)+\int_{b}^{u(t)} \frac{\sqrt{m} a^{2}(\eta) g(\eta)}{G(\eta)} d \eta\right) \leq 0 .
$$

Therefore,

$$
\limsup _{t \rightarrow+\infty}\left(\int_{b}^{u(t)}\left(\frac{a^{2}(\eta) F(\eta) g(\eta)}{(2 G(\eta))^{\frac{3}{2}}}+\frac{\sqrt{m} a^{2}(\eta) g(\eta)}{G(\eta)}\right) d \eta+\frac{h(v(t))}{\sqrt{2 G(u(t))}}\right)<\infty .
$$


Since, $h(v(t))>F(u(t))$ and $u(t) \rightarrow+\infty$ as $t \rightarrow+\infty$, (2.7) contradicts (2.5). This contradiction completes the proof.

Corollary 2.1. Assume (2.1) and (2.2) hold and $h(y) \leq m y$, for $y<0$, with $|y|$ sufficiently large and some $m>0$. Then system (1.1) has property $\left(X^{+}\right)$in the right half-plane if

$$
\liminf _{x \rightarrow+\infty} \frac{F(x)}{\sqrt{2 G(x)}}>-\infty
$$

and

$$
\limsup _{x \rightarrow+\infty} \int_{b}^{x}\left(\frac{a^{2}(\eta) F(\eta) g(\eta)}{(2 G(\eta))^{\frac{3}{2}}}+\frac{\sqrt{m} a^{2}(\eta) g(\eta)}{G(\eta)}\right) d \eta=+\infty
$$

for some $b>0$.

ExAmple 2.1. Consider system (1.1) with $F(x)=-3 \sqrt{m} x+2.2 \sqrt{m} x \sin ^{2}(x)$, $g(x)=x, a(x)=1$ and $h(y)=m y$, with $m>0$. We have

$$
\liminf _{x \rightarrow+\infty} \frac{1}{F(x)} \int_{b}^{x} \frac{g(\xi)}{F(\xi)} d \xi \leq \lim _{n \rightarrow+\infty} \frac{\int_{b}^{2 n \pi} \frac{1}{-3+2.2 \sin ^{2}(x)}}{-6 n m \pi}=\frac{\int_{0}^{2 \pi} \frac{1}{-3+2.2 \sin ^{2}(x)}}{-6 m \pi}<\frac{1}{4 m},
$$

for every $b>0$. Thus, condition (1.6) is not satisfied and Theorem $\mathrm{C}$ is inapplicable; however,

$$
\begin{aligned}
& \lim _{x \rightarrow+\infty} \int_{b}^{x}\left(\frac{a^{2}(\eta) F(\eta) g(\eta)}{(2 G(\eta))^{\frac{3}{2}}}+\frac{\sqrt{m} a^{2}(\eta) g(\eta)}{G(\eta)}\right) d \eta \\
& =\sqrt{m} \lim _{x \rightarrow+\infty} \int_{b}^{x} \frac{-s+2.2 s \sin ^{2}(s)}{s^{2}} d s \\
& =\sqrt{m} \lim _{x \rightarrow+\infty} \int_{b}^{x} \frac{0.1-1.1 \cos (2 s)}{s} d s=+\infty,
\end{aligned}
$$

and therefore, by Corollary 2.1 system (1.1) has property $\left(X^{+}\right)$in the right half-plane.

EXAMPLE 2.2. Consider system (1.1) with $F(x)=-k \sqrt{m} x+l \sqrt{m} x \sin ^{2}(x), g(x)=$ $x, a(x)=1$ and $h(y)=m y$ with $k+\frac{l}{2}>2$ and $m>0$. We have

$$
\begin{aligned}
& \lim _{x \rightarrow+\infty} \int_{b}^{x}\left(\frac{a^{2}(\eta) F(\eta) g(\eta)}{(2 G(\eta))^{\frac{3}{2}}}+\frac{\sqrt{m} a^{2}(\eta) g(\eta)}{G(\eta)}\right) d \eta \\
& =\lim _{x \rightarrow+\infty} \sqrt{m} \int_{b}^{x} \frac{k-2+l \sin ^{2}(s)}{s} d s \\
& =\lim _{x \rightarrow+\infty} \sqrt{m} \int_{b}^{x} \frac{\left(k-2+\frac{l}{2}\right)-\frac{l}{2} \cos (2 s)}{s} d s=+\infty .
\end{aligned}
$$

Therefore, by Corollary 2.1 system (1.1) has property $\left(X^{+}\right)$in the right half-plane.

Corollary 2.2. Assume (2.1) and (2.2) hold and $h(y) \leq m y$, for $y<0$, with $|y|$ sufficiently large and some $m>0$, and $G(+\infty)=+\infty$. Then system (1.1) has property 
$\left(X^{+}\right)$in the right half-plane if

$$
\liminf _{x \rightarrow+\infty} \frac{F(x)}{\sqrt{2 G(x)}}>-2 \sqrt{m} .
$$

Proof. Suppose that

$$
\liminf _{x \rightarrow+\infty} \frac{F(x)}{\sqrt{2 G(x)}}>\lambda>-2 \sqrt{m}
$$

then there exists a $b>0$ such that

$$
F(x) \geq \lambda \sqrt{2 G(x)} \text { for } x>b .
$$

Thus, we have

$$
\begin{aligned}
& \limsup _{x \rightarrow+\infty} \int_{b}^{x}\left(\frac{a^{2}(\eta) F(\eta) g(\eta)}{(2 G(\eta))^{\frac{3}{2}}}+\frac{\sqrt{m} a^{2}(\eta) g(\eta)}{G(\eta)}\right) d \eta \\
& \geq \limsup _{x \rightarrow+\infty} \int_{b}^{x}\left(\frac{(\lambda+2 \sqrt{m}) a^{2}(\eta) g(\eta)}{2 G(\eta)}\right) d \eta \\
& =\limsup _{x \rightarrow+\infty} \frac{\lambda+2 \sqrt{m}}{2}(\ln G(x)-\ln G(b))=+\infty
\end{aligned}
$$

Hence, by Corollary 2.1 system (1.1) has property $\left(X^{+}\right)$in the right half-plane.

REMARK 2.1. For $a(x)=1$ and $h(y)=y$, Corollary 2.2 gives the classical result of Filippov (condition (1.3)); also Theorem 2.1, Corollary 2.1 and Corollary 2.2 give Theorem 2.3, Corollary 2.5 and Corollary 2.6 in [1], respectively.

THEOREM 2.2. Assume (2.1) and (2.2) hold, $F(x)$ is differentiable for $x>0$, with $x$ sufficiently large, and $h(y) \leq$ my for $y<0$, with $|y|$ sufficiently large. Then system (1.1) has property $\left(\mathrm{X}^{+}\right)$in the right half-plane if

$$
\limsup _{x \rightarrow+\infty}\left(\int_{b}^{x} \frac{F^{\prime}(\eta)}{\sqrt{2 G(\eta)}} d \eta+\sqrt{m} \ln G(x)\right)=+\infty,
$$

for some $b>0$.

Proof. We can write

$$
\begin{aligned}
\frac{F(x)}{\sqrt{2 G(x)}} & =\int_{b}^{x}\left(\frac{F(\eta)}{\sqrt{2 G(\eta)}}\right)^{\prime} d \eta+\frac{F(b)}{\sqrt{2 G(b)}} \\
& =\int_{b}^{x}\left(\frac{F^{\prime}(\eta)}{\sqrt{2 G(\eta)}}-\frac{a^{2}(\eta) g(\eta) F(\eta)}{(\sqrt{2 G(\eta)})^{\frac{3}{2}}}\right) d \eta+\frac{F(b)}{\sqrt{2 G(b)}} .
\end{aligned}
$$

Therefore, (2.9) is equivalent to (2.5) and the proof is complete.

EXAmple 2.3. Assume $G(+\infty)=+\infty$ and $m>0$. Consider system (1.1) with

$$
F(x)=-2 \sqrt{2 m G(x)}+K(\sqrt{G(x)}),
$$


where

$$
\int^{\infty} \frac{K^{\prime}(\eta)}{\eta} d \eta=\infty, \text { and } \lim _{u \rightarrow \infty} K^{\prime}(u)=0
$$

(for example $K(u)=\int_{a}^{u} \frac{d t}{\ln t}, a>0$ ). Also assume that $h(y) \leq m y$, for $y<0$, with $|y|$ sufficiently large. Then using L'Hôpital's rule we get

$$
\begin{aligned}
& \lim _{x \rightarrow+\infty} \frac{1}{F(x)} \int_{b}^{x} \frac{a^{2}(\eta) g(\eta)}{F(\eta)} d \eta=\lim _{x \rightarrow+\infty} \frac{a^{2}(x) g(x)}{F^{\prime}(x) F(x)} \\
& =\lim _{x \rightarrow+\infty} \frac{1}{\left(-2 \sqrt{2 m}+\frac{K(\sqrt{G(x)})}{\sqrt{G(x)}}\right)\left(-\sqrt{2 m}+\frac{K^{\prime}(\sqrt{G(x))}}{2}\right)}=\frac{1}{4 m}<\frac{\beta}{m},
\end{aligned}
$$

for every $\beta>1 / 4$. Hence, Theorem $\mathrm{C}$ is inapplicable to this example. However,

$$
\begin{aligned}
& \limsup _{x \rightarrow+\infty} \int_{b}^{x} \frac{F^{\prime}(\eta)}{\sqrt{2 G(\eta)}} d \eta+\sqrt{m} \ln G(x) \\
& \quad=\limsup _{x \rightarrow+\infty} \int_{b}^{x} \frac{a^{2}(\eta) g(\eta) K^{\prime}(\sqrt{G(\eta)})}{2 \sqrt{2} G(\eta)} d \eta=\limsup _{x \rightarrow+\infty} \frac{1}{\sqrt{2}} \int_{b}^{x} \frac{K^{\prime}(\eta)}{\eta} d \eta=+\infty .
\end{aligned}
$$

Thus, by Theorem 2.2 system (1.1) has property $\left(X^{+}\right)$in the right half-plane.

LEMMA 2.3. Suppose that $F_{1}(x) \leq F_{2}(x)$ for $x>0$ sufficiently large and system (1.1) with $F_{1}$ has property $\left(X^{+}\right)$in the right half-plane; then system (1.1) with $F_{2}$ has property $\left(X^{+}\right)$in the right half-plane, too.

Proof. Suppose that system (1.1) with $F_{2}$ fails to have property $\left(X^{+}\right)$in the right half-plane. Then there exists a positive semi-trajectory of (1.1) starting at a point $\left(u_{0}, v_{0}\right)$ with $u_{0}>0$ and $h\left(v_{0}\right)>F_{1}\left(u_{0}\right)$, which does not meet the vertical isocline $h(v)=$ $F_{2}(u)$. This trajectory can be regarded as the graph of a function $v=\psi_{2}(u)$ which is a solution of

$$
\frac{d y}{d x}=\frac{-a^{2}(x) g(x)}{h(y)-F(x)} .
$$

Let $v=\psi_{1}(u)$ be the graph of the solution of system (1.1) corresponding to $F_{1}$, such that $(u(0), v(0))=\left(u_{0}, v_{0}\right)$. We can assume that $u_{0}$ is sufficiently large; thus, we have

$$
\psi_{1}^{\prime}(u)=\frac{-a^{2}(u) g(u)}{h(v)-F_{1}(u)} \geq \frac{-a^{2}(u) g(u)}{h(y)-F_{2}(u)}=\psi_{2}^{\prime}(u), \quad \text { for } u \geq u_{0} .
$$

Hence, $\quad \psi_{1}(u) \geq \psi_{2}(u)>F_{2}(u) \geq F_{1}(u), \quad$ for $u \geq u_{0}$. Therefore, system (1.1) corresponding to $F_{1}$ fails to have property $\left(X^{+}\right)$in the right half-plane. This contradiction completes the proof.

The proof of the following theorem follows directly from Lemma 2.3 and the result of Example 2.3.

TheOREM 2.3. Assume $G(+\infty)=+\infty$ and $h(y) \leq m y$ for some $m>0$ and $y<0$, with $|y|$ sufficiently large. Moreover,

$$
F(x) \geq-2 \sqrt{2 m G(x)}+K(\sqrt{G(x)}),
$$


where

$$
\int^{\infty} \frac{K^{\prime}(\eta)}{\eta} d \eta=\infty \text { and } \lim _{u \rightarrow \infty} K^{\prime}(u)=0
$$

Then system (1.1) has property $\left(X^{+}\right)$in the right half-plane.

Corollary 2.3. Assume (2.1) and (2.2) hold and $h \in C^{1}(-\infty, \alpha)$, for some $\alpha \in \mathbb{R}$, $h^{\prime}(-\infty)=+\infty$ and $G(+\infty)=+\infty$. Then system (1.1) has property $\left(X^{+}\right)$in the right half-plane if

$$
\liminf _{x \rightarrow+\infty} \frac{F(x)}{\sqrt{2 G(x)}}>-\infty
$$

Proof. Choose $m>0$ sufficiently large such that (2.8) holds. Since, $h^{\prime}(-\infty)=+\infty$, there exists an $M<0$ such that

$$
h^{\prime}(y)>2 m \text { for } y<M<0
$$

hence,

$$
h(y)-h(M)=(y-M) h^{\prime}(\xi)<2 m(y-M),
$$

where $y<\xi<M$. Thus,

$$
h(y)<m y,
$$

for $y<0$, with $|y|$ sufficiently large. Therefore, Corollary 2.2 implies that system (1.1) has property $\left(X^{+}\right)$in the right half-plane.

Now consider system (1.1) with $h(y)=\lambda|y|^{p} \operatorname{sgn}(y)$. This case has been considered by many authors $[\mathbf{5}, \mathbf{1 4}]$. We have the following result for this case.

Corollary 2.4. Assume (2.1) and (2.2) hold, $h(y)=\lambda|y|^{p} \operatorname{sgn}(y)$, with $\lambda>0$ and $p>1$, and $G(+\infty)=+\infty$. Then system (1.1) has property $\left(X^{+}\right)$in the right half-plane if

$$
\limsup _{x \rightarrow+\infty} \frac{|F(x)|}{\sqrt{2 G(x)}}<\infty
$$

Notice that when $h$ is differentiable, in (2.4) we can let $K(y)=h(y)$. Hence, we have the following result under the assumption $h \in C^{1}(-\infty, \alpha)$, for some $\alpha \in \mathbb{R}$. The proof is similar to that of Theorem 2.1; so we omit it.

Theorem 2.4. Assume (2.1) and (2.2) hold, $h \in C^{1}(-\infty, \alpha)$, for some $\alpha \in \mathbb{R}$, $h(-\infty)=-\infty$ and $h^{\prime}(y)$ is increasing for $y<0$, with $|y|$ sufficiently large. Then system (1.1) has property $\left(X^{+}\right)$in the right half-plane if

$$
\limsup _{x \rightarrow+\infty}\left(\int_{b}^{x}\left(\frac{a^{2}(\eta) F(\eta) g(\eta)}{(2 G(\eta))^{\frac{3}{2}}}+\frac{\sqrt{h^{\prime}\left(h^{-1}(F(\eta))\right)} a^{2}(\eta) g(\eta)}{G(\eta)}\right) d \eta+\frac{F(x)}{\sqrt{2 G(x)}}\right)=+\infty,
$$

for some $b>0$. 
Corollary 2.5. Assume (2.1) and (2.2) hold, $h \in C^{1}(-\infty, \alpha)$, for some $\alpha \in \mathbb{R}$, $h(-\infty)=-\infty, h^{\prime}(y)$ is increasing for $y<0$, with $|y|$ sufficiently large, and $G(+\infty)=$ $+\infty$. Then system (1.1) has property $\left(X^{+}\right)$in the right half-plane if there exists an $\alpha>0$ such that

$$
\frac{F(x)}{2 \sqrt{2 G(x)}}+\sqrt{h^{\prime}\left(h^{-1}(F(\eta))\right)}>\alpha,
$$

for $x>0$ sufficiently large.

REMARK 2.2. Notice that if $h^{\prime}(y)$ is increasing on $(-\infty, \alpha)$ for some $\alpha \in \mathbb{R}$, then $0 \leq m=\lim _{y \rightarrow-\infty} h^{\prime}(y)$ exists. Hence, if $m \neq 0$, we have $h(y) \leq m y$ and $h^{-1}(y) \geq \frac{y}{m}$, for $y<0,|y|$ sufficiently large. Therefore, when it is difficult to compute $h^{-1}(F(x))$ we can replace it in (2.10) and (2.11) by $\frac{F(x)}{m}$.

Corollary 2.6. Assume (2.1) and (2.2) hold, $h(y)=\lambda|y|^{p} \operatorname{sgn}(y)$, with $\lambda>0$ and $p \geq 1$. Then system (1.1) has property $\left(X^{+}\right)$in the right half-plane if there exists an $\alpha>0$ such that

$$
\frac{F(x)}{2 \sqrt{2 G(x)}}>-\sqrt{p} \lambda^{\frac{1}{2 p}}(|F(x)|)^{\frac{p-1}{2 p}}+\alpha,
$$

for $x>0$ sufficiently large.

COROLlaRY 2.7. Assume (2.1) and (2.2) hold and $h(y)=\lambda|y|^{p} \operatorname{sgn}(y)$, with $\lambda>0$ and $p \geq 1$. Then system (1.1) has property $\left(X^{+}\right)$in the right half-plane if there exists a $\beta>1$ such that

$$
|F(x)| \leq \beta(8 p)^{\frac{p}{p+1}} \lambda^{\frac{1}{p+1}}(G(x))^{\frac{p}{p+1}},
$$

for $x>0$, sufficiently large.

3. Sufficient conditions for property $\left(Y^{+}\right)$. In this section we present some sufficient conditions for system (1.1) to have property $\left(Y^{+}\right)$in the right half-plane. Throughout this section we assume that there exists a $\delta>0$,

$$
F(x)>0 \text { for } 0<x<\delta,
$$

because if $F(x)$ has an infinite number of positive zeros clustering at $x=0$, then obviously system $(1.1)$ has property $\left(Y^{+}\right)$. Now suppose that there exists a solution $(u(t), v(t))$ of $(1.1)$ which starts at a point $\left(u_{0}, v_{0}\right)$ with $F\left(u_{0}\right)=h\left(v_{0}\right)$ and whose graph remains in the region $\{(u, v): u \geq 0$ and $h(v)<F(u)\}$ for all future time. Taking the vector field of (1.1) into account, we see that if the positive semi-trajectory of this solution crosses the $x$-axis, then it also meets the negative $y$-axis. Since $\dot{u}<0$ in this region, we have

$$
\lim _{t \rightarrow \infty} u(t)=\lim _{t \rightarrow \infty} v(t)=0
$$

so, we may assume that $u_{0}$ is sufficiently small and

$$
0<u(t) \leq u_{0} \text { for } t>0 \text {. }
$$


Now let

$$
\chi(t)=\int_{b}^{u(t)} \frac{a^{2}(s) F(s) g(s)}{(2 G(s)) \frac{3}{2}} d s+\frac{K(v(t))}{\sqrt{2 G(u(t))}},
$$

where $K(u) \leq h(u)$ and $K^{\prime}(u) \geq 0$ for $u>0$ sufficiently small; then

$$
\begin{aligned}
\dot{\chi}(t)= & \dot{u}(t) \frac{a^{2}(u(t)) F(u(t)) g(u(t))}{(2 G(u(t))) \frac{3}{2}} d s \\
& -\frac{2 a(u(t)) g(u(t)) G(u(t)) K^{\prime}(v(t))+a^{2}(u(t)) g(u(t)) K(v(t)) \dot{u}(t)}{(2 G(u(t))) \frac{3}{2}} \\
\leq & -\frac{2 a(u(t)) g(u(t)) G(u(t)) K^{\prime}(v(t))+a^{3}(u(t)) g(u(t))(\dot{u}(t))^{2}}{(2 G(u(t))) \frac{3}{2}} \\
\leq & \frac{a^{2}(u(t)) g(u(t)) \sqrt{K^{\prime}(v(t))}}{G(u(t))} \dot{u}(t) .
\end{aligned}
$$

Hence,

$$
\dot{\chi}(t) \leq \frac{a^{2}(u(t)) g(u(t)) \sqrt{K^{\prime}(v(t))}}{G(u(t))} \dot{u}(t)
$$

for $t>0$ sufficiently large.

THEOREM 3.1. Assume that $h$ is differentiable and $h(y) \geq m y$, for $y>0$ sufficiently small and some $m>0$. Then system (1.1) has property $\left(Y^{+}\right)$in the right half-plane if

$$
\liminf _{x \rightarrow 0^{+}}\left(\int_{x}^{b}\left(\frac{a^{2}(\eta) F(\eta) g(\eta)}{(2 G(\eta))^{\frac{3}{2}}}-\frac{\sqrt{m} a^{2}(\eta) g(\eta)}{G(\eta)}\right) d \eta\right)=-\infty,
$$

for some $b>0$.

Proof. We prove the theorem by contradiction. Suppose that system (1.1) fails to have property $\left(Y^{+}\right)$in the right half-plane. Then from (3.2) we have

$$
\frac{d}{d t}\left(\chi(t)-\int_{b}^{u(t)} \frac{\sqrt{m} a^{2}(\eta) g(\eta)}{G(\eta)} d \eta\right) \leq 0,
$$

for $t>0$ sufficiently large. Therefore,

$$
\limsup _{t \rightarrow 0^{+}}\left(\int_{b}^{u(t)}\left(\frac{a^{2}(\eta) F(\eta) g(\eta)}{(2 G(\eta))^{\frac{3}{2}}}-\frac{\sqrt{m} a^{2}(\eta) g(\eta)}{G(\eta)}\right) d \eta+\frac{h(v(t))}{\sqrt{2 G(u(t))}}\right)<\infty .
$$

Since, $\frac{h(v(t))}{\sqrt{2 G(u(t))}}>0$ and $u(t) \rightarrow 0$ as $t \rightarrow+\infty$, (3.5) contradicts (3.3). This contradiction completes the proof.

EXAMPLE 3.1. Consider system (1.1) with $F(x)=2 x-x^{3}, g(x)=x, h(y)=y$ and $a(x)=1$. Then

$$
\lim _{x \rightarrow 0^{+}} \frac{1}{F(x)} \int_{0}^{x} \frac{g(\eta)}{F(\eta)} d \eta=\lim _{x \rightarrow 0^{+}} \frac{\int_{0}^{x} \frac{d \eta}{2-\eta^{2}} d \eta}{2 \eta-\eta^{3}}=\frac{1}{4}
$$


Thus, conditions (1.6) and (1.7) are not satisfied. However,

$$
\liminf _{x \rightarrow 0^{+}} \int_{x}^{b}\left(\frac{\left(2 \eta-\eta^{3}\right) \eta}{\eta^{3}}-\frac{2 \eta}{\eta^{2}}\right) d \eta=\liminf _{x \rightarrow 0^{+}} \int_{x}^{b}-\frac{1}{\eta} d \eta=-\infty .
$$

Hence, Theorem 3.1 implies that system (1.1) has property $\left(Y^{+}\right)$.

The following results can be proved from Theorem 3.1, similar to the results presented in Section 2.

COROLlary 3.1. Assume that $h(y) \geq m y$, for $y>0$ sufficiently small and some $m>0$. Then system (1.1) has property $\left(Y^{+}\right)$in the right half-plane if

$$
\limsup _{x \rightarrow 0^{+}} \frac{F(x)}{\sqrt{2 G(x)}}<2 \sqrt{m} .
$$

COROLlary 3.2. Assume that $h^{\prime}(0)=+\infty$. Then system (1.1) has property $\left(Y^{+}\right)$in the right half-plane if

$$
\limsup _{x \rightarrow 0^{+}} \frac{F(x)}{\sqrt{2 G(x)}}<\infty
$$

THEOREM 3.2. Assume that $h^{\prime}(y)$ is decreasing for $y>0$ sufficiently small. Then system (1.1) has property $\left(\mathrm{Y}^{+}\right)$in the right half-plane if

$$
\liminf _{x \rightarrow 0^{+}}\left(\int_{x}^{b}\left(\frac{a^{2}(\eta) F(\eta) g(\eta)}{(2 G(\eta))^{\frac{3}{2}}}-\frac{\sqrt{h^{\prime}\left(h^{-1}(F(x))\right)} a^{2}(\eta) g(\eta)}{G(\eta)}\right) d \eta\right)=-\infty,
$$

for some $b>0$.

COROllary 3.3. Assume that $h^{\prime}(y)$ is decreasing for $y>0$ sufficiently small. Then system (1.1) has property $\left(Y^{+}\right)$in the right half-plane if there exists an $\alpha<0$ such that

$$
\frac{F(x)}{2 \sqrt{2 G(x)}}-\sqrt{h^{\prime}\left(h^{-1}(F(x))\right)}<\alpha,
$$

for $x>0$ sufficiently small.

COROLlary 3.4. Assume $h(y)=\lambda|y|^{p} \operatorname{sgn}(y)$, with $\lambda>0$ and $p<1$. Then system (1.1) has property $\left(Y^{+}\right)$in the right half-plane if there exists an $\alpha<0$ such that

$$
\frac{F(x)}{2 \sqrt{2 G(x)}}<\sqrt{p} \lambda^{\frac{1}{2 p}}(F(x))^{\frac{p-1}{2 p}}+\alpha
$$

for $x>0$ sufficiently small.

COROllary 3.5. Assume $h(y)=\lambda|y|^{p} \operatorname{sgn}(y)$, with $\lambda>0$ and $p<1$. Then system (1.1) has property $\left(Y^{+}\right)$in the right half-plane if there exists $a<1$ such that

$$
F(x) \leq \beta(8 p)^{\frac{p}{p+1}} \lambda^{\frac{1}{p+1}}(G(x))^{\frac{p}{p+1}},
$$

for $x>0$ sufficiently small. 


\section{REFERENCES}

1. A. Aghajani and A. Moradifam, Some sufficient conditions for the intersection with the vertical isocline in the Liînard plane, Appl. Math. Lett. 19 (2006), 491-497.

2. A. Aghajani and A. Moradifam, Intersection with the vertical isocline in the Liénard plane, Nonlin. Anal. 68 (2007), 3475-3484.

3. A. Aghajani and A. Moradifam, On the homoclinic orbits of the generalized Liénard equations, Appl. Math. Lett. 20 (2007), 345-351.

4. A. Aghajani and A. Moradifam, Oscillation of solutions of second-order nonlinear differential equations of Euler type, J. Math. Anal. Appl. 326 (2007), 1076-1089. $26-39$.

5. C.-M. Ding, The homoclinic orbits in the Liînard plane, J. Math. Anal. Appl. 191 (1995),

6. A. F. Filippov, A sufficient condition for the existence of a stable limit cycle for an equation of the second order, Mat. Sb. (N.S.) 30 (1952), 171-180.

7. J. R. Graef, On the generalized Liénard equation with negative damping, J. Diff. Eq. 12 (1972), 34-62.

8. M. Gyllenberg and Y. Ping, The generalized Liénard systems, Discrete Cont. Dyn. Syst. 8 (2002), 1043-1057.

9. T. Hara, Notice on the Vinograd type theorems for Liénard system, Nonlin. Anal. 22 (1994), 1437-1443.

10. T. Hara and J. Sugie, When all trajectories in the Liénard plane cross the vertical isocline? Nonlin. Diff Eq. Appl. 2(4) (1995), 527-551.

11. T. Hara and T. Yoneyama, On the global center of generalized Liénard equation and its application to stability problems, Funkcial. Ekvac. 28 (1985), 171-192.

12. M. Hesaaraki and $A$. Moradifam, Intersection with the vertical isocline in the generalized Liénard equations, J. Math. Anal. Appl. 334 (2007), 787-798.

13. M. W. Hirsch and S. Smale, Differential equations, dynamical systems, and linear algebra (Academic Press, New York, 1974).

14. J. F. Jiang, The global stability of a class of second order differential equations, Nonlin. Anal. 28 (1997), 855-870.

15. J. P. LaSale and S. Lefschetz, Stability by Liapunov's direct method (Academic Press, New York, 1961).

16. S. Lefschetz, Differential equations: Geometric theory (Dover, New York, 1977). 1964).

17. G. Sansone and R. Conti, NonLinear differential equations, Macmillan (New York,

18. J. Sugie, Homoclinic orbits in generalized Liénard systems, J. Math. Anal. Appl. 309 (2005), 211-226. 83-97.

19. J. Sugie, Liénard dynamics with an open limit orbit, Nonlin. Diff. Eq. Appl. 8 (2001),

20. J. Sugie and T. Hara, Existence and non-existence of homoclinic trajectories of the Liénard system, Discrete Cont. Dyn. Syst. 2 (1996), 237-254.

21. G. Villari and F. Zanolin, On a dynamical system in the Liénard plane: Necessary and sufficient conditions for the intersection with the vertical isocline and applications, Funkcial. Ekvac. 33 (1990), 19-38.

22. P. Yan and J. F. Jiang, Concerning the center of the generalized Liénard systems, J. Syst. Sci. Math. Scis. 19 (1999), 353-358.

23. Z. F. Zhang, T. R. Ding, W. Z. Huang and Z. X. Dong, Qualitative theory of ordinary differential equations, Translation of Mathematics Monographs vol. 101 (American Mathematical Society, Providence, RI, 1992).

24. Y.-R. Zhou and X.-R. Wang, On the conditions of a center of the Liénard equation, J. Math. Anal. Appl. 180 (1993), 43-59. 